\title{
Salt effect in post-synthesis hydrothermal treatment of MCM-41
}

\author{
Hong-Ping Lin ${ }^{\text {a }}$, Chung-Yuan Mou ${ }^{b, *}$ \\ ${ }^{a}$ Institute of Chemistry, Academia Sinica, Nankang, Taipei 115, Taiwan \\ ${ }^{\mathrm{b}}$ Department of Chemistry and Center of Condensed Matter Research, National Taiwan University, 1 Roosevelt Road Section 4, \\ Taipei 106, Taiwan
}

Received 24 January 2002; received in revised form 6 May 2002; accepted 7 May 2002

\begin{abstract}
Post-synthesis hydrothermal treatment of MCM-41 mesoporous silica provides a convenient method for pore expansion and silica wall thickening for improvement of its stability. The physical chemistry of the process is investigated by examining the effects of water content, salts and aluminum on pore expansion. A hydrothermal treatment at $150{ }^{\circ} \mathrm{C}$ in water or a salt solution leads to controlled pore expansion. The pore size and wall thickness vary with the kind of anion of the salt and their concentrations. The salt effect follows the well-known binding strength of the Hofmeister series of anion for the cationic surfactant, $\mathrm{NO}_{3}^{-}>\mathrm{Br}^{-}>\mathrm{Cl}^{-}>\mathrm{SO}_{4}^{2-} \sim \mathrm{F}^{-}$. It is proposed that an equilibrium of distributing surfactants inside the MCM-41 channels and in solution controls the pore size and wall thickness upon varying the salts. The anion $\left(\mathrm{X}^{-}\right)$binds with cationic surfactant molecules $\left(\mathrm{S}^{+}\right)$in solution to shift the equilibrium of surfactant/silicate binding leading to less surfactant and water in the pore, and hence less pore expansion. The effect of ammonia hydrothermal treatment is to shift the equilibrium to stronger surfactant/silicate binding and thus more pore expansion. At neutral condition, the wall thickness varies inversely with respect to the pore diameter. The wall thickness variation agrees with a model of elastic deformation of the wall silica materials at high temperature.
\end{abstract}

(c) 2002 Elsevier Science Inc. All rights reserved.

Keywords: Pore expansion; Hydrothermal treatment; Salt effect; MCM-41

\section{Introduction}

Since its discovery [1], M41S material has attracted wide attention for its rich variety of synthetic strategies for modifying its structure and morphology. Considerable efforts were made to vary its synthesis process in order to control its

\footnotetext{
${ }^{*}$ Corresponding author. Tel.: +886-2-33665251; fax: +886-223660954.

E-mail address: cymou@ccms.ntu.edu.tw (C.-Y. Mou).
}

structure order [2], pore size [3] and morphology $[4,5]$. The processes are complicated by many factors involving surfactant self-assembly in solution [6], mesophases transformation [7], surface functionalization [8] and hydrothermal re-structuring of silica $[9,10]$.

Post-synthesis hydrothermal re-structuring of MCM-41 materials is a particularly useful technique for metal incorporation [11], adjusting structural order [12], pore expansion [13-16] and enhancing hydrothermal stability $[16,17]$. However, 
due to the richness of experimentally controllable variables, the mechanism of hydrothermal restructuring is not yet well understood. Factors important in hydrothermal treatment are temperature [10,18], $\mathrm{pH}$ value [2,19], and additives $[17,20]$. They affect detailed structural characters such as wall-thickness [21], degree of silica condensation [16] and pore sizes [13-16]. Different experimental variables affect structural characters in different ways. In recent literature, there are rich combinations of adjusting these variables. The synthesis situation can be rather confusing if one wants also a clear understanding of the physical chemistry behind the various treatments.

Post-synthesis hydrothermal treatments have been developed for various purposes. The problems are varied, such as wall structure, hydrothermal stability or pore size control. Among these problems, the pore expansion appears to be most amenable to a well-controlled study. The pore size is a well-defined physical quantity, and its change can be followed in a systematical way.

Moreover, pore-size control is important in applications such as catalysis, adsorption and confinement of nanomaterials. For example, in catalytic applications, larger pore system could lead to less pore-blocking and higher conversion [22]. Quantum confinement of semiconductor in the nanochannels of mesoporous silica requires fine control of the pore size of the host [23]. In this paper, we discuss the pore-expansion problem by post-synthesis hydrothermal treatment in a wellcontrolled approach such that individual variable can be examined in more focused way.

Khushalani et al. [9] were the first to report the effect of post-synthesis hydrothermal treatment at $150{ }^{\circ} \mathrm{C}$ on MCM-41 materials in mother solution leading to large pore expansion up to $7 \mathrm{~nm}$ (more than $100 \%$ ). They attribute the pore expansion to a significant penetration of water into the pore during treatment. Stucky and coworkers [6] used postsynthesis hydrothermal treatment to expand pore of MCM-41, and they found they could expand the pore by about $30 \%$ at $100{ }^{\circ} \mathrm{C}$ for geminal surfactants with divalent head groups. It should be noted that water instead of mother liquor was used in the postsynthesis hydrothermal treatment. Corma et al. [14] used synthesis gel in a high temperature $\left(150^{\circ} \mathrm{C}\right)$ hydrothermal treatment and obtained large expansion of pore similar to that of Khushalani et al. In a similar post-synthesis hydrothermal treatment of MCM-41, Cheng et al. [13a,b] found a moderate pore size increase accompanied by a large increase of the wall-thickness. In the work of Chen et al. [16] water was used as medium in hydrothermal treatment and they also found a mild pore expansion with improved stability. Recently Kruk, Jaroniec, Sayari (KJS) and coworkers [10,15,24,25] initiated a detailed attack on the pore expansion problem of post-synthesis restructuring of MCM41. They used a one-step high temperature hydrothermal treatment in mother liquor. They proposed that the main driving force for the large pore expansion is the in situ decomposition of the cationic surfactant and the generation of dimethylhexadecylamine (DMHA) which acts as a powerful expander [13a,25]. It seems both the hydroxide ion and the quaternary ammonium cations such as $\mathrm{TMA}^{+}$could act as catalysts for the thermal decomposition of the surfactant $\mathrm{C}_{16} \mathrm{TMAB}$. In fact quaternary ammonium cations are used in many of the case of large expansion.

Summarizing observations from previous works, it seems that there are two kinds of aqueous medium used for hydrothermal pore expansion leading to different extent of pore expansions. If one uses mother liquor as the hydrothermal medium, the high $\mathrm{pH}$ condition would activate surfactant decomposition and help silica hydrolysis. The silica undergoes extensive restructuring with large neutral expander DMHA solubilized in the hydrophobic core of the surfactant organization. On the other hand, pore expansion in water (thus lower $\mathrm{pH}$ condition) usually leads to a minor expansion by about $30 \%$ and more complete siloxane bond condensation. This often leads to better structural order and stability. Here the driving force and the mechanism are not completely clear. However, recently Tolbert and coworkers [7] speculated that the silica annealing process, driven by the changes in surfactant conformation, is responsible for the minor pore expansion of MCM41 in water at high temperature. Furthermore, they showed the same driving force could also lead to consecutive interesting phase transformations of the silica/surfactant composites under hydro- 
thermal treatment [7]. The silica framework can respond to changes in surfactant organization under hydrothermal conditions.

Since the surfactant system in the synthesis is lyotropic in nature, instead of changing the temperature there is actually another way to change the surfactant equilibrium in the system. It is by varying the electrolytes in the solution composition, in particular the counterion $\mathrm{X}^{-}$species. In the synthesis of MCM-41, the $\mathrm{S}^{+} \mathrm{I}^{-}$binding has to compete against the simple counterion $\mathrm{X}^{-}[6]$. Previously we have shown the factors involved in determining the mesostructure formation are: counterion adsorption equilibrium of $\mathrm{X}^{-}$on micellar surface and catalyzed silica condensation near micellar surface [26]. There is a specific effect following the well-known Hofmeister series [27] in surfactant-anion binding strength. We have shown that the specific counterion binding of $\mathrm{X}^{-}$could influence the formation of the mesostructure both in acid synthesis [26] and in alkaline synthesis [28] of mesoporous silica.

In this paper, we study the pore expansion under hydrothermal conditions with neutral aqueous solutions of simple salts. There are two purposes underlying this study. First, we are interested in finding out whether there is a specific order, as in Hofmeister series, in changing the pore size and wall thickness. We will gain insight toward a better understanding of the silica restructuring in response to the surfactant system in the surfactant-silica composite materials perturbed by the salts. Secondly, it is known that in the hydrothermal treatment of MCM-41 in salt solutions [17,29,30], the structure and stability (corresponding to silica wall thickness) of the materials are markedly improved. The reinforced MCM-41 would be useful in many catalytic applications [31]. In this method the surfactant-counterion binding is important in controlling the pore structure and hydrothermal stability.

\section{Experimental}

\subsection{Materials}

Sodium silicate $\left(27 \% \mathrm{SiO}_{2}, 14 \% \mathrm{NaOH}\right.$, Aldrich) was used as the silica source. Quaternary ammonium surfactants are cetyltrimethylammonium bromide $\left(\mathrm{C}_{16} \mathrm{H}_{33}\left(\mathrm{CH}_{3}\right)_{3} \mathrm{NBr} ; \mathrm{C}_{16} \mathrm{TMAB}\right)$ from Acrôs. The source of aluminum was sodium aluminate $\mathrm{NaAlO}_{2}$ (Riede-de Haën). Sulfuric acid was from Merck. The sodium salts $(\mathrm{NaX} ; \mathrm{X}=\mathrm{F}$, $\mathrm{Cl}, \mathrm{Br}, \mathrm{NO}_{3}, \mathrm{~S}_{1 / 2} \mathrm{O}_{2}$ ) were from Acrôs or Janssen Chimica. All these materials were used directly without further purification.

\subsection{Synthetic procedure}

\subsubsection{Preparation of room temperature-made MCM-41 mesoporous silicas and aluminosilicates samples}

To prepare as-synthesized mesoporous pure silica MCM-41 materials, the delayed neutralization process we previously reported was used [32]. First of all, sodium silicate was combined with a clear surfactant aqueous solution under stirring, and then a gel mixture was formed. After mixing for about $10-20 \mathrm{~min}$ at $32{ }^{\circ} \mathrm{C}$, a suitable amount of $1.20 \mathrm{M} \mathrm{H}_{2} \mathrm{SO}_{4}$ was added into the gel mixture, and $\mathrm{pH}$ value of the reaction compositions was adjusted to about 9.0. The molar ratio of the resulting gel composition is: $1 \mathrm{C}_{16} \mathrm{TMAB}: 1 \mathrm{SiO}_{2}$ : $0.39 \mathrm{Na}_{2} \mathrm{O}: 0.29 \mathrm{H}_{2} \mathrm{SO}_{4}:(50-400) \mathrm{H}_{2} \mathrm{O}$. The aluminosilicate MCM-41 was synthesized with the same process except for adding a proper amount of $\mathrm{NaAlO}_{2}(\mathrm{Si} / \mathrm{Al}=37)$ into the $\mathrm{C}_{16} \mathrm{TMAB}$ solution of surfactant-sodium silicate. The as-synthesized solid products were recovered by filtration, washed with a large amount of deionized water such that $\mathrm{pH}$ of the wash liquid was less than 8 and then the samples were dried in air at $100{ }^{\circ} \mathrm{C}$.

\subsubsection{Postsynthesis hydrothermal treatment on RT- made mesoporous materials}

The $1.0 \mathrm{~g}$ dried RT-made mesoporous samples were added into $50.0 \mathrm{~g} \mathrm{H}_{2} \mathrm{O}$, and the $\mathrm{pH}$ value of the suspension was about $8-9$. Then, the material was loaded and sealed in an autoclave at 100 or $150{ }^{\circ} \mathrm{C}$ for three days. For comparing the salt effect on the pore expansion and wall thickening, different sodium salts with desired amounts are combined with the solution before hydrothermal treatment. To examine the influence of the $\mathrm{pH}$ value on the lattice expansion, an ammonia solution was used instead of water. For studying water 
content effect on pore expansion, a desired amount of RT-made sample $(0.25-3.0 \mathrm{~g})$ was added in $50 \mathrm{~g}$ $\mathrm{H}_{2} \mathrm{O}$ solution. The final product was regained by filtration and washing. The as-synthesized samples were calcined in air at 560 or $580{ }^{\circ} \mathrm{C}$ for $6 \mathrm{~h}$ to remove the organic species occluded in the nanochannels of the MCM-41 aluminosilicate.

\subsection{Characterization}

X-ray powder diffraction (XRD) patterns were taken on a Scintag X1 diffractometer with $\mathrm{Cu} \mathrm{K}_{\alpha}$ radiation $(\lambda=0.154 \mathrm{~nm})$. The $\mathrm{N}_{2}$ adsorption-desorption isotherms were collected at $77 \mathrm{~K}$ on a Micromeritics ASAP 2010 apparatus. Prior to the analysis, the sample was outgassed at $300{ }^{\circ} \mathrm{C}$ for about $6 \mathrm{~h}$ in $10^{-3}$ Torr. The pore size distribution was obtained from the analysis of the adsorption branch of the isotherms using the BJH (BarrettJoyner-Halenda) method. The solid state ${ }^{29} \mathrm{Si}$ MAS NMR experiments were performed at room temperature on a Brueker MSL 300 or 500 NMR spectrometer with a magnetic field of 6.6 or $11 \mathrm{~T}$ respectively. The surfactant content of the mesoporous silicas was measured by thermogravimetric analysis (TGA). TGA were conducted with a ULVAC TGA-7000RH thermogravimetric system. In a typical experiment, $\approx 50 \mathrm{mg}$ of sample was heated to $700{ }^{\circ} \mathrm{C}$ at $10{ }^{\circ} \mathrm{C} / \mathrm{min}$ under dry air. The surfactant content was determined from the weight loss between the temperature range of $150-450{ }^{\circ} \mathrm{C}$. Higher weight loss in this range corresponds to higher surfactant content in the as-synthesized surfactant-silica mesostructural samples.

\section{Results}

In this section, we report on the phenomenon of pore expansion after hydrothermal treatment at $150{ }^{\circ} \mathrm{C}$ with various conditions. These variations include the ratio of sample to water, salt addition and aluminum incorporation on the framework. They affect the pore size and wall-thickness in a systematic way that can be understood. We present the results in this section. In the next section, we then discuss the mechanism underlining the

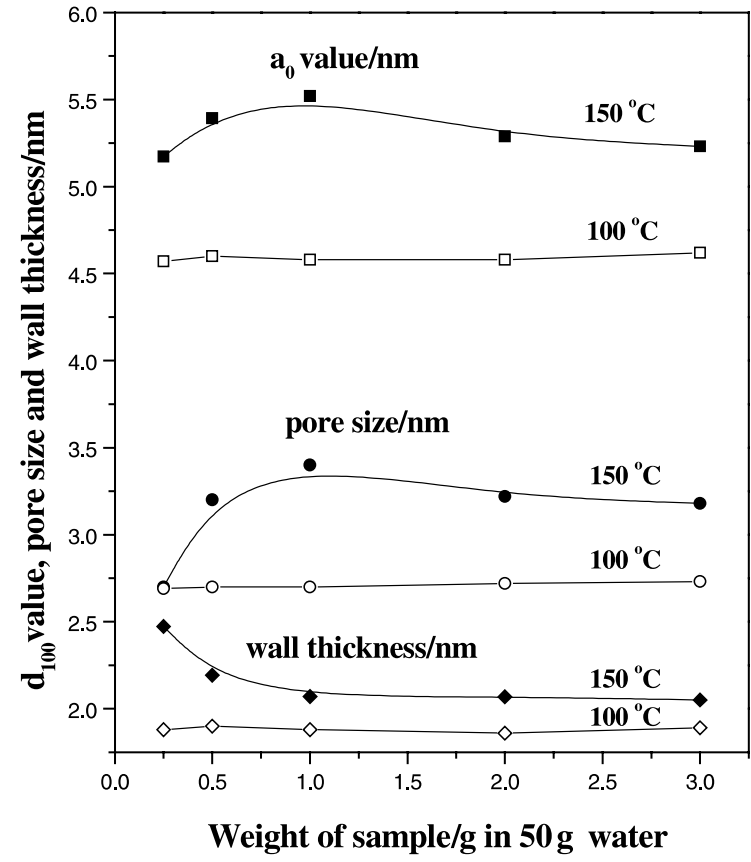

Fig. 1. Plots of $d_{100}$ value, pore size, and wall thickness of the mesoporous silica synthesized from $\mathrm{C}_{16}$ TMAB-silicate- $\mathrm{H}_{2} \mathrm{O}$ after hydrothermal treatment for three days. The horizontal axis expresses the ratio of sample weight to $50 \mathrm{~g}$ water.

re-structuring and further data in support of our interpretations.

Fig. 1 presents the structural data of the MCM41 samples after hydrothermal treatment at 100 and $150{ }^{\circ} \mathrm{C}$ for three days. They include the pore size and the nearest neighbor channel distance $a_{0}$ $\left(2 d_{100} / \sqrt{ } 3\right)$. The wall thickness is the difference between the above two sizes. We varied the amount of water used in the treatment. The $x$-axis is the weight of sample to $50 \mathrm{~g}$ of water used.

First we see that the data for the $100{ }^{\circ} \mathrm{C}$ condition, which is usually applied in normal procedure, show constant sizes, independent of the sample/water ratio. Secondly as one increases the temperature to $150{ }^{\circ} \mathrm{C}$ in treatment, there are modest increases in both the pore size (from 2.7 up to $3.3 \mathrm{~nm}$ ) and the wall thickness (from 1.7 to 2.5 $\mathrm{nm})$. The pore expansions at $150{ }^{\circ} \mathrm{C}$ now depend on the sample to water ratio $(r)$. As $r$-value increases, wall thickness decreases while the pore diameter increases to a constant value. Apparently, the temperature of $100{ }^{\circ} \mathrm{C}$ is too low for 
obvious mesostructure annealing to occur. It has been shown previously [7] that the onset temperature for annealing is $\mathrm{pH}$ dependent. In Ref. [7], the reported onset temperature is between 90 and $60{ }^{\circ} \mathrm{C}$ for alkaline solution (in $0.15-0.235 \mathrm{M}$ $\mathrm{NaOH}$ ). Our solution $\mathrm{pH}$ is much closer to neutral and annealing onset should be more difficult. Therefore mesostructure annealing is not considerably expected at $100^{\circ} \mathrm{C}$. At $150^{\circ} \mathrm{C}$, there is more extensive restructuring. Both the pore size and wall thickness increase compared to those for the 100 ${ }^{\circ} \mathrm{C}$ cases. However, we also note that, when comparing across the sample/water ratio at the same temperature of $150{ }^{\circ} \mathrm{C}$, the pore size change is in the opposite direction of the wall thickness trend.

We next performed the hydrothermal treatment in salt solution of the as-synthesis MCM-41 composite at $150{ }^{\circ} \mathrm{C}$ for three days at the fixed sample/ water weight ratio of $1 / 50$. The results for the size changes are shown in Fig. 2. We note that the case for the ammonia solution is in different trend from

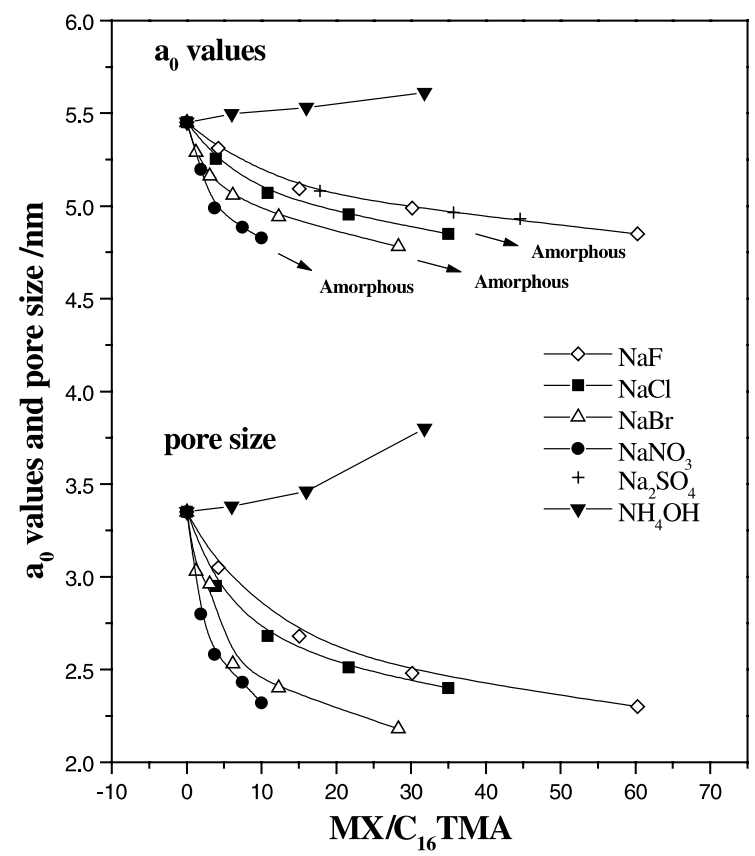

Fig. 2. The unit cell size $\left(a_{0}\right)$ and pore size for the mesoporous silica after hydrothermal treatment at $150{ }^{\circ} \mathrm{C}$ for three days, plotted as functions of salt to surfactant $\left\{(\mathrm{MX}) / \mathrm{C}_{16} \mathrm{TMAB}\right\}$ ratio. The weight ratio of the as-synthesized sample to water is fixed at $1 / 50$. the rest of the salt solutions. As the concentration of ammonia is increased, the pore size also increases while the wall-thickness decreases. Previously, we have used ammonia solution in the hydrothermal treatment of acid-synthesized mesoporous silica [33]. It is a convenient method for structure improvement under the desired $\mathrm{pH}$ value conditions.

For the other NaX salt solutions, Fig. 2 shows that as the concentration of counterion $\mathrm{X}^{-}$is increased, the pore size decreases while the wallthickness increases. The slope of the decrease of pore size is anion-specific (the cation matters little) and strength of the influence follows the order of $\mathrm{NO}_{3}^{-}>\mathrm{Br}^{-}>\mathrm{Cl}^{-}>\mathrm{F}^{-}$. This is the same lyotropic (Hofmeister) series that has been reported for many other hydrophobic or amphiphilic solution organizations. This is also found in our previous investigation of the counterion effect in the kinetics of formation of surfactants-silica mesoporous materials [26,28].

The decrease in pore size upon the increase of salt concentration is accompanied by a gradual decrease in the structure order of the hexagonal channels. An example for the case of $\mathrm{NaBr}$ solution is shown in the XRD patterns in Fig. 3. The concentration of $\mathrm{NaBr}$ increases from A to F. Kim et al. [30] observed also a gradual loss of structure order in their study of salt effect in hydrothermal treatment. They attributed this to a branching of micelles, which causes a transition from hexagonal mesophase to a disordered network.

Fig. 3 also shows that the $d$-spacing of the hexagonal phase decreases as the salt concentration increases. But the hexagonal phase order cannot persist indefinitely. The point at the highest salt concentration in Fig. 2 is in fact the end point for the existence of mesostructure beyond which the pores collapse and form amorphous silica. This point of structure collapse seems to occur at the pore size about $2.2 \mathrm{~nm}$ for all the salts examined. Moreover, the salt concentration for the mesostructures collapse also decreased as Hofmeister series.

The results in Figs. 1-3 are for pure silica MCM-41. When aluminum is incorporated into the structural framework, we found that the expansion of the pore size is very difficult in a $150^{\circ} \mathrm{C}$ 


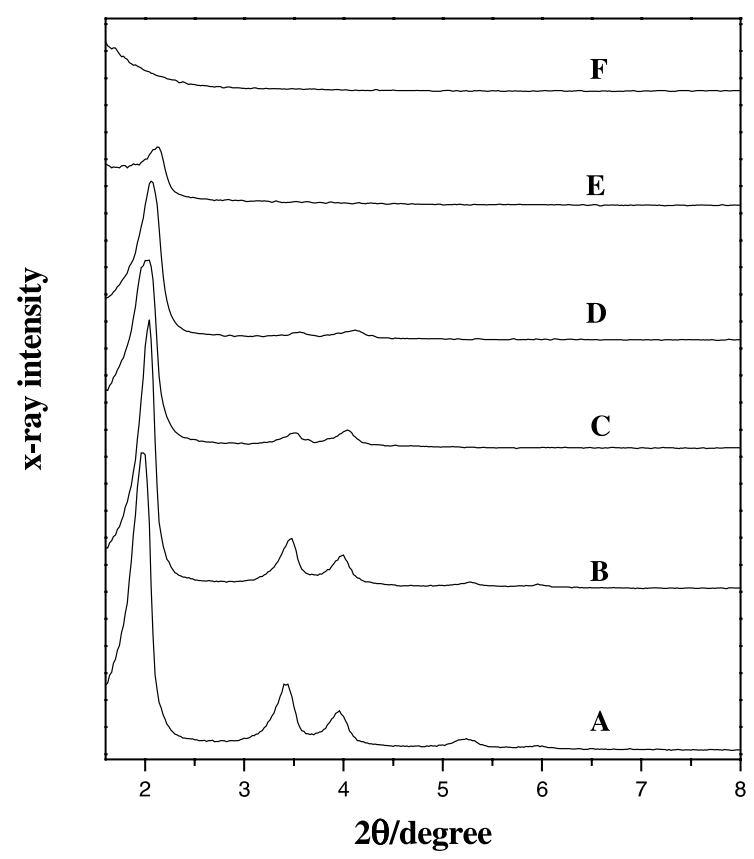

Fig. 3. XRD patterns of the mesoporous samples after $150{ }^{\circ} \mathrm{C}$ hydrothermal treatment for three days in $\mathrm{NaBr}$ solution. The concentration of $\mathrm{NaBr}$ increases from $\mathrm{A}$ to $\mathrm{F}$. (A) $\mathrm{NaBr} / \mathrm{C}_{16} \mathrm{TMAB}=0$; (B) $\mathrm{NaBr} / \mathrm{C}_{16} \mathrm{TMAB}=3.07$; (C) $\mathrm{NaBr} /$ $\mathrm{C}_{16} \mathrm{TMAB}=12.3 ; \quad$ (D) $\mathrm{NaBr} / \mathrm{C}_{16} \mathrm{TMAB}=28.3$; (E) $\mathrm{NaBr} /$ $\mathrm{C}_{16} \mathrm{TMAB}=48.0 ; \quad$ (F) $\mathrm{NaBr} / \mathrm{C}_{16} \mathrm{TMAB}=60.0$. The weight ratio of the as-synthesized sample to water is fixed at $1 / 50$.

hydrothermal treatment. Fig. 4 shows a comparison of the pore size for the two cases, with and without aluminum incorporation. When aluminum is present $(\mathrm{Si} / \mathrm{Al}=37)$, curve $\mathrm{A}$ shows a capillary condensation occurs at $P / P_{0}$ about 0.32 0.33 corresponding to a pore size distribution between $2.40-2.70 \mathrm{~nm}$, close to the sizes of the samples synthesized at $100{ }^{\circ} \mathrm{C}$. However, when no aluminum is present, the pore was swollen after $150{ }^{\circ} \mathrm{C}$ hydrothermal treatment (Fig. 4B), compared to the MCM-41 samples at $100{ }^{\circ} \mathrm{C}$ or without any hydrothermal treatment. The capillary change of $P / P_{0}$ occurs at about 0.42 corresponding to a pore size of $3.4 \mathrm{~nm}$ (see also Fig. 1).

\section{Discussion}

There are two questions about the pore size and wall thickness change we would like to focus in

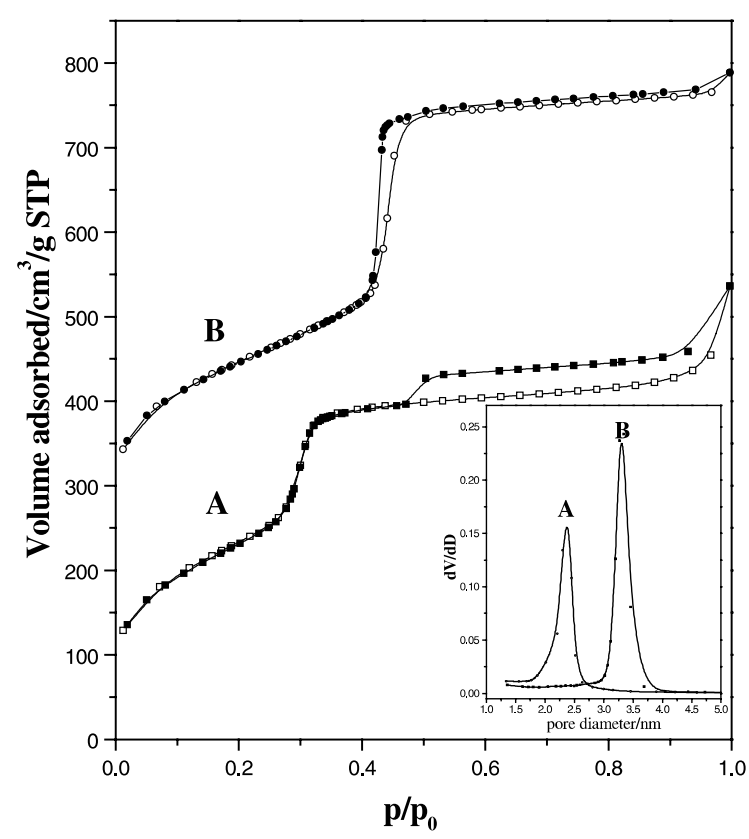

Fig. 4. The $\mathrm{N}_{2}$ adsorption-desorption isotherms of the pure silica and aluminosilicate $(\mathrm{Si} / \mathrm{Al}=37)$ mesoporous materials after $150{ }^{\circ} \mathrm{C}$ hydrothermal post-treatment for three days. The inserted plot is the pore size distribution curves of these samples. (A) $\mathrm{Si} / \mathrm{Al}=37$; (B) $\mathrm{Si} / \mathrm{Al}=\infty$. The weight ratio of the assynthesized sample to water solution is $1 / 50$.

this section. The first is the question of the thermodynamic driving force for pore expansion. And the second is concerned about the way by which silica is reconstructed in hydrothermal treatment.

From literatures, we can summarize that there are basically two kinds of driving force for pore size change upon hydrothermal treatment of silica: one is the change of silica surface condensation and the other is the change of surfactant organization. It has been long known that porous silica (without template) would expand its pore upon hydrothermal treatment [34]. When porous silica spheres were prepared by an application of the sol-gel method and a hydrothermal treatment of the hydro-gel [35], the pore-radius increased with the hydrothermal temperature and the duration of the treatment. Here water penetration into the pore probably depends on the surface free energy. The more negative curvature favors hydrogen bonding between surface silanol groups. At higher 
temperature the dehydration would make siloxane (hydrophobic) group which favors lower curvature. For the as-synthesized surfactant/silica mesocomposite system, Khushalani et al. [9] proposed water penetration as the cause of hydrothermal pore expansion. On the other hand, organics solubilized inside the micellar channel can also swell the channel. It has been proposed that the main driving force for the large pore expansion is the in situ decomposition of the cationic surfactant and the generation of DMHA which penetrated into the hydrophobic core of surfactants, and acts thus as an expander [13a,25]. The silica wall respond to the increase of organics by reconstructing around it.

The second question we are interested in is how the silica reconstruction is accomplished. Is the reconstruction a local adjustment of the silica network or there is extensive dissolution/deposition? How is the wall-thickness adjusted to the pore change? The addition of salt in the aqueous medium of hydrothermal treatment allows us a whole range of controlled pore size. We observed in Fig. 2 a systematic inverse relationship between the pore-size and the wall-thickness. We would like to understand the relationship.

\subsection{Pore expansion under hydrothermal treatment in water}

For understanding the surfactant-silicas mesostructural restructuring after the $150{ }^{\circ} \mathrm{C}$ hydrothermal treatment, the TGA instrument was used to investigate the surfactant retention weight. The ${ }^{29} \mathrm{Si}$ MAS NMR directly analyzed the local bonding of silica in the as-synthesized $150{ }^{\circ} \mathrm{C}$ hydrothermally treated samples. Fig. 5 shows the organic surfactant $/ \mathrm{SiO}_{2}$ ratios and the $\mathrm{Q}^{3} / \mathrm{Q}^{4}\left(\mathrm{Q}^{3}\right.$ : $\left.(\mathrm{SiO})_{3} \mathrm{Si}-\mathrm{OH} ; \mathrm{Q}^{4}:(\mathrm{SiO})_{4} \mathrm{Si}\right)$ ratios as a function of the sample weight to $50 \mathrm{~g}$ water. One can clearly find that the surfactant $/ \mathrm{SiO}_{2}$ ratios dramatically decreased after $150{ }^{\circ} \mathrm{C}$ hydrothermal treatment (the surfactant $/ \mathrm{SiO}_{2}$ of the starting material is 0.91). In addition, the surfactant $/ \mathrm{SiO}_{2}$ ratio of the $150{ }^{\circ} \mathrm{C}$ hydrothermally treated sample increase with the sample weight to water. It was suggested that some part of the surfactants were extracted from the mesoporous channels, and the removal

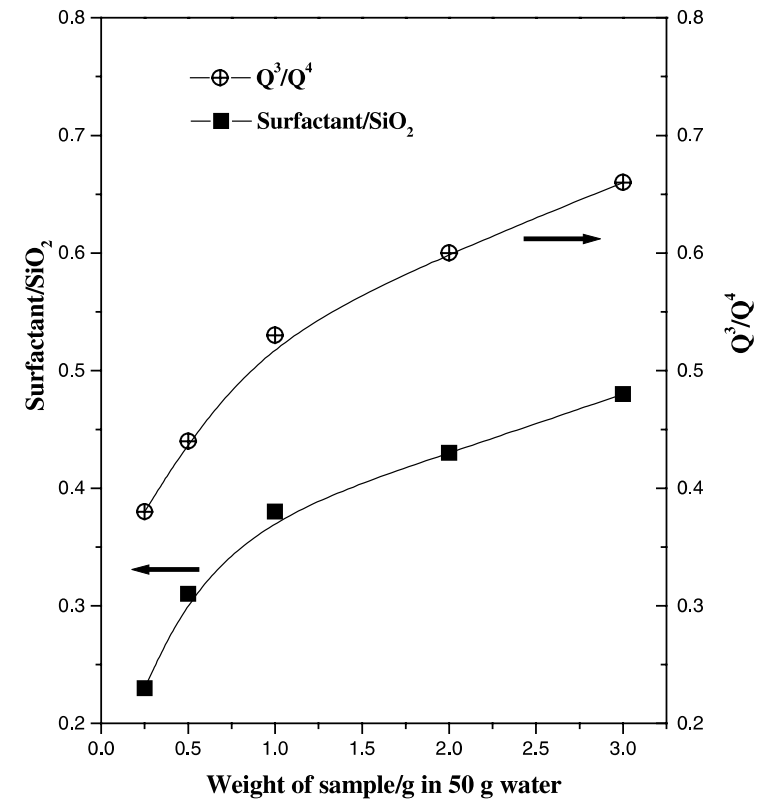

Fig. 5. The surfactant/SiO 2 and $\mathrm{Q}^{3} / \mathrm{Q}^{4}$ ratios of the as-synthesized MCM-41 mesoporous materials before and after hydrothermal at $150{ }^{\circ} \mathrm{C}$ for three days plotted as a function of sample weight in $50 \mathrm{~g}$ water.

amount reasonably increased with the water contents. Similar to the trend of the surfactant $/ \mathrm{SiO}_{2}$ ratio, the $\mathrm{Q}^{3} / \mathrm{Q}^{4}$ ratio corresponding to the silica condensation also increased with the sample weight to $50 \mathrm{~g}$ water that indicated the silica further condensed as the increase of water content. Combining these results, a conclusion should be drawn as the mechanism of the surfactant amount change in the surfactant-silicas mesostructured composites. Let us consider the following equilibrium involving the surface siloxane condensation and the change of charge balance.

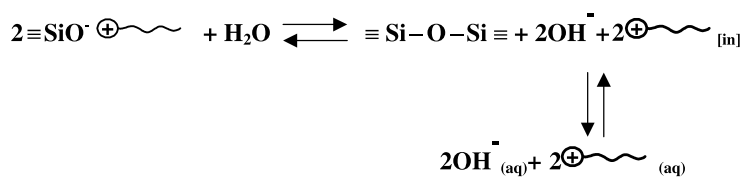

One can see that surfactant molecules can move out of the silicate/surfactant composite due to decreased surface charge density of the silica 
wall after some siloxane formation under high temperature $150{ }^{\circ} \mathrm{C}$ condition. Eq. (1) also indicates the equilibrium will shift towards the right hand side when there is more water in the system and, therefore, one expect that less surfactant retention in the silicate/surfactant mesostructural sample.

For comparison, we also analyzed the surfactant weight retention and $\mathrm{Q}^{3} / \mathrm{Q}^{4}$ ratio (surfactant/ $\mathrm{SiO}_{2}=0.76 ; \mathrm{Q}^{3} / \mathrm{Q}^{4}=0.88$ ) for the as-synthesized sample after $100{ }^{\circ} \mathrm{C}$ hydrothermally treated sample. The results for the $100{ }^{\circ} \mathrm{C}$ hydrothermally treated sample are roughly independent of the sample/water ratio.

The samples for the $150{ }^{\circ} \mathrm{C}$ treatment are the same set of samples where their size data are shown in Fig. 1. The higher surfactant retention is favored at higher MCM-41 materials/water ratio. From Fig. 5, for $150{ }^{\circ} \mathrm{C}$ treated samples, one can see that the more is the surfactant content, the less silica condensation. Up to sample to water ratio $1 / 50$, this is also parallel with concomitant increase of pore size. However if the two samples at 100 and $150{ }^{\circ} \mathrm{C}$ (at the same sample to water ratio $1 / 50$ ) are compared, the lower temperature sample results in higher surfactant/silica ratio but gives a smaller pore (Fig. 1). This is due to a thinner wall at $100{ }^{\circ} \mathrm{C}$. Upon increasing temperature, the simultaneous increase of wall thickness, pore size and the decrease of surfactant content indicate some space filling and surfactant replacing substance penetrate the pore after high temperature treatment. Quite likely, this is water penetration as proposed by Khushalani et al. [9]. The silica condensation reaction would favor a larger pore and provide the driving force for the water penetration. This kind of pore-expansion is, however, quite modest by increasing about $0.7 \mathrm{~nm}$. The larger expansion reported in the literature is most likely due to surfactant decomposition to amine in alkaline condition.

\subsection{Counterion effect under hydrothermal treatment in salt solution}

Let us consider a similar equilibrium for the surfactant removal by counterion binding as shown in Eq. (2):

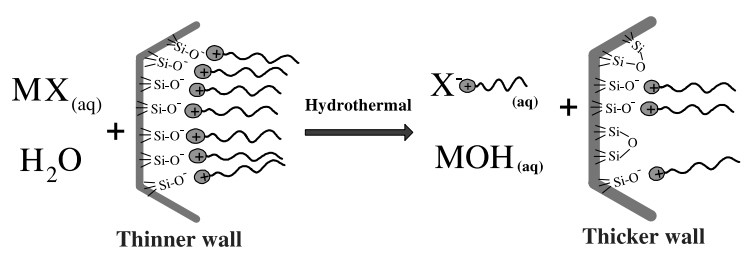

(2)

When salt (MX) is present in the hydrothermal solution, the equilibrium will be shifted to the right and there will be loss of surfactant from the composite due to the competitive effect between $\mathrm{X}^{-}$and negative-charged silica wall to the cationic surfactants. This is what we see in Fig. 2 that the pore size of the calcined sample decreases with the increase of salt concentration but the wall thickness increases. Besides, different anions will differ in their binding strength to the cationic surfactant. The order in counterion binding is well known as the Hofmeister series:

$\mathrm{NO}_{3}^{-}>\mathrm{Br}^{-}>\mathrm{Cl}^{-}>\mathrm{SO}_{4}^{2-} \sim \mathrm{F}^{-}$

This is the order we observe in the decrease of pore-size in Fig. 2. This order is also observed in our previous study of the counterion effect in the kinetics of formation of mesoporous silica $[26,28]$. There are many other parallel observations. For example, recent results obtained from direct EMF measurement of degree of counterion association to cationic surfactant also follow the above lyotropic order [36]. To examine the effect of the cations $\left(\mathrm{M}^{+}\right)$of the salts, four kind chloride compounds (such as $\mathrm{LiCl}, \mathrm{NaCl}, \mathrm{KCl}$ and $\left(\mathrm{CH}_{3}\right)_{4} \mathrm{Cl}$ ) were used to test. From the results (Table 1), we readily conclude that the cationic species do not

Table 1

The physical properties of the MCM-41 mesoporous materials after $150{ }^{\circ} \mathrm{C}$ hydrothermal treatment in different chloride salts water solution

\begin{tabular}{llllll}
\hline Salts $^{\mathrm{a}}$ & $\begin{array}{l}d_{100} \\
(\mathrm{~nm})\end{array}$ & $\begin{array}{l}a_{0} \\
(\mathrm{~nm})\end{array}$ & $\begin{array}{l}\text { BET } \\
\text { S.A. } \\
\left(\mathrm{m}^{2} / \mathrm{g}\right)\end{array}$ & $\begin{array}{l}\text { Pore } \\
\text { size } \\
(\mathrm{nm})\end{array}$ & $\begin{array}{l}\text { Wall } \\
\text { thickness } \\
(\mathrm{nm})\end{array}$ \\
\hline $\mathrm{LiCl}$ & 4.32 & 4.98 & 560 & 2.50 & 2.48 \\
$\mathrm{NaCl}$ & 4.29 & 4.95 & 567 & 2.55 & 2.40 \\
$\mathrm{KCl}$ & 4.36 & 5.03 & 587 & 2.53 & 2.50 \\
$\left(\mathrm{CH}_{3}\right)_{4} \mathrm{NCl}$ & 4.33 & 5.00 & 574 & 2.52 & 2.48 \\
\hline
\end{tabular}

${ }^{\mathrm{a}}$ The ratio of the salts/ $\mathrm{C}_{16} \mathrm{TMA}$ is about 20.0 . 
have obvious influence on the equilibrium of Eq. (2). In other words, the addition of salts is not a simple ion exchanging reaction, but a surfactant competing and silica condensation reaction.

One should note here that at $150{ }^{\circ} \mathrm{C}$ the pore contraction (c.f. Fig. 2), upon hydrothermal treatment in salt solutions, probably also exclude the occurrence of surfactant decomposition to DMHA. If DMHA was produced, one would observe some swelling action leading to pore expansion instead of contraction here. As the solution $\mathrm{pH}$ is in neutral condition, the base-catalyzed Hoffmann elimination reaction probably did not occur. We thus only observe surfactant removal upon hydrothermal reaction in salt solution.

As more surfactant molecules are removed from the surfactants-silica composite as increasing the salt concentration, it would be more difficult to maintain the hexagonal order of the MCM-41 materials. Thus, we observe in Fig. 3 that the structure order becomes poorer and finally it leads to structure collapse to amorphous silica at high salt concentration.

When there is aluminum incorporation in the framework, the wall becomes negatively charged. It leads to stronger binding to the cationic surfactant and reduces the amount of the removed surfactant during hydrothermal treatment [37]. The weaker anion-surfactant binding cannot compete against the aluminum effect. The pore size is more difficult to change compared to the pure siliceous MCM-41 as shown in Fig. 4.

\subsection{Pore expansion under hydrothermal treatment in ammonia solution}

We next discuss the hydrothermal treatment in ammonia solution. Previously, we have used ammonia solution in the hydrothermal treatment of acid-synthesized mesoporous silica [33]. It is a convenient method for structure improvement.

The result in pore size change by the addition of ammonia is opposite of the other cases of salt solution. As the concentration of ammonia is increased, the pore size increases while the wallthickness decreases. We have also performed TGA analysis of the ammonia treated samples at various concentrations. Fig. 6 gives the weight retentions

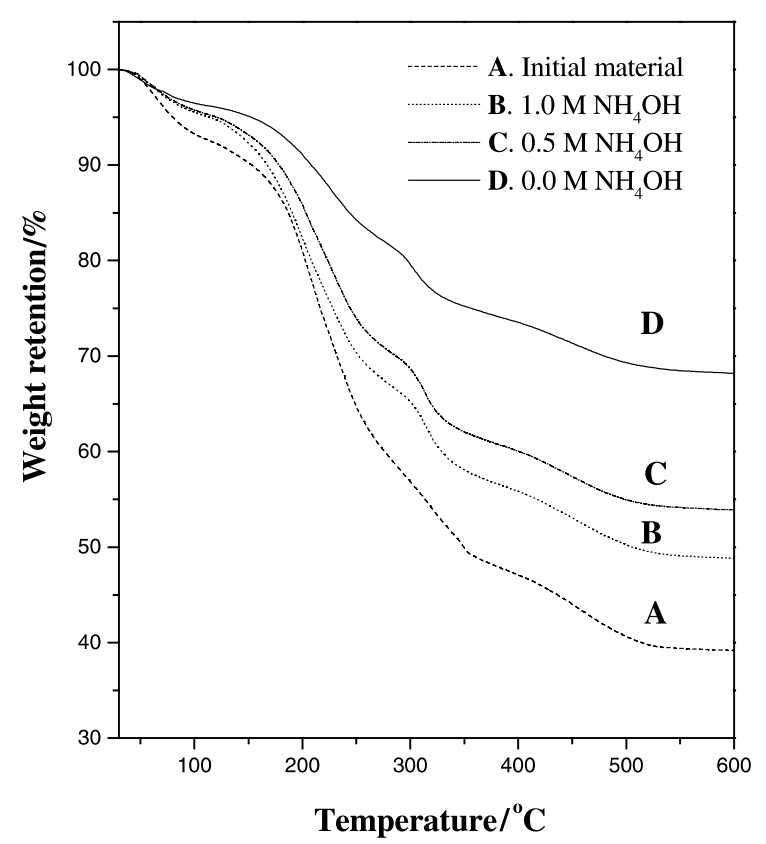

Fig. 6. TGA curves for the mesoporous materials after hydrothermal treatment at $150{ }^{\circ} \mathrm{C}$ for three days in ammonium solution of different concentrations, where the weight ratio of the as-synthesized sample to ammonia solution is fixed at $1 / 50$.

as function of temperature. We see that the higher the ammonia concentration, the more the surfactant retention. From the equilibrium reaction (1), we can expect that the reaction will shift to the left in alkaline condition and thus lead to retention of surfactant. More surfactant retained in the MCM41 products of the larger pore. However, since this is in alkaline condition, we cannot rule out the Hoffmann reaction leading to the expander DMHA.

\subsection{The conservation of channels}

We have qualitatively observed the pore change at $150{ }^{\circ} \mathrm{C}$, upon the addition of electrolyte, is due to the changes in the equilibrium (1). Surfactant removal due to counterion binding would shrink the pore relative to the treatment in pure water. However, silica wall also should play some role. We would like to know how the silica responds to the change in surfactant removal. We see, from 


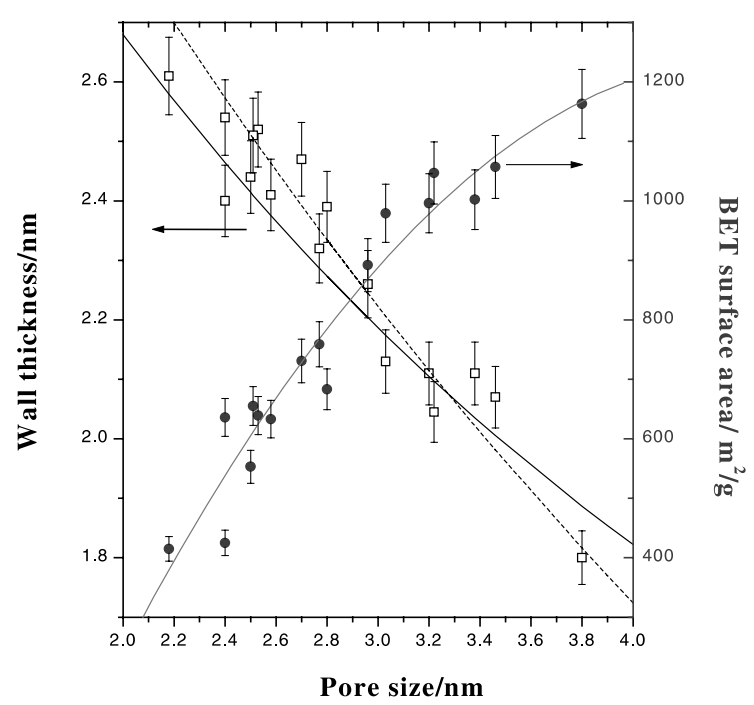

Fig. 7. The wall thickness and BET surface area for the mesoporous silica materials after hydrothermal post-treatment at $150{ }^{\circ} \mathrm{C}$ for three days under various reactions conditions plotted as a function of pore size. The dash line and dot line are the results calculated according to Eqs. (3) and (4) for the hexagonal-shaped and circle-like pore respectively and assuming number of nanochannels within mesoporous materials being conserved.

Fig. 2 that the changes of wall thickness are in an opposite trend from the pore size change. We plot the data in Fig. 2 in another way as in Fig. 7. The wall thickness is the difference between $a_{0}$ and pore diameter value. Although the data are somewhat scattered, we can see the clear inverse relationship between pore size and wall thickness. Also we plotted the BET surface area data against pore size. When pore size is increased, the BET surface area increases as expected. Since we treated hydrothermally the sample under neutral $\mathrm{pH}$ condition, we do not expect there is much silica dissolution into the solution. So the silica materials should be conserved in the sample. The question is then how the silica/surfactant composites reorganize themselves to adjust to the surfactant changes. We model the change by making a few assumptions. First, we assume the structural adjustment is only local; e.g. no extensive dissolution-deposition is assumed. The system simply changes the pore and wall locally while it maintains the hexagonally arranged mesostructure. Secondly, we assume the number of channels and
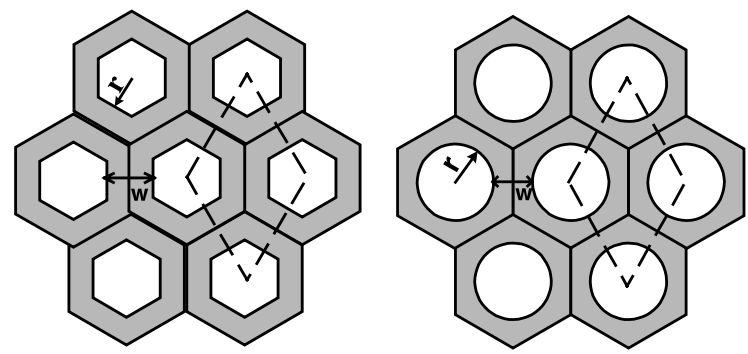

Fig. 8. The schematic diagrams of the hexagonal and cylindrical-shaped nanopore models. The $r$ and $w$ indicate the radius and the wall thickness of the nanochannels, respectively. The diamond dot line area is an unit cell of the mesostructure.

their lengths are conserved, e.g. no loss of channels. This seems to be a reasonable assumption since we have not observed changes in morphology. Thirdly, for the channel shape we assume two models: the hexagon and the circular. They are shown in Fig. 8. The shaded areas in Fig. 8 represent the solid materials of silica wall. Now if the pore contracts to a smaller value, the conservation of channels and silica would allow us to predict how the wall thickness would change. One can calculate the relationship between pore size and wall thickness according to Eqs. (3) and (4) for the two models.

For hexagonal channels

$w=-2 r+\sqrt{4 r^{2}+2 A / \sqrt{3}}$

For cylindrical channels

$w=-2 r+\sqrt{2 \pi r^{2} / \sqrt{3}+2 A / \sqrt{3}}$

$A$ is the area of silica in a unit cell.

The results are plotted in Fig. 7. The solid curve is based on hexagonal channel model and the dot curve is based on cylinder channel. Amazingly, the experimental results follow the model prediction quite closely. The scattering of the data does not allow us to distinguish which model is better however. For BET surface area data however, we did not attempt a fit based on theoretical model because there are contributions due to surface area from texture and micro-porosity so it is uncertain the extent of contribution of mesopores.

The close agreement between the predicted and experimental results with just one-parameter fit 
leads us to propose that the channels are preserved in hydrothermal reconstruction. The adjustment of wall thickness follows the change in the surfactant organization continuously; that is, the silica adjusts locally to preserve symmetry and volume. This is corroborated by several observations in this laboratory and others reported in the literature. It has been reported the morphology of the MCM-41 materials does not change much after hydrothermal treatment [30,38]. Previously, we have reported a synthesis of tubular form of MCM-41 [38]. The morphology is basically fixed at the stage of as-synthesized composite, prolonged hydrothermal treatment even at the higher temperature of $150{ }^{\circ} \mathrm{C}$ does not transform them into particular form or other morphology. Gross et al., in a study on the phase stability of periodic silica/surfactant nano-structured composites driven by temperature increase, reported continuous adjustment of silica framework subjecting to the change in surfactant conformation [7].

We should note here that in determining the pore size of the MCM-41 materials we have used the standard $\mathrm{BJH}$ method based on Kelvin equation of capillary condensation. Many researchers challenged the use of $\mathrm{BJH}$ method claiming it under-estimate the pore size [38,39]. Among the newer methods proposed, the one based on geometrical construction was investigated intensively by Kruk et al. [38].

If one shifts to other methods, what would be changed in Fig. 7? It has been shown by KJS that their new geometrical method can be reproduced by using a corrected BJH method. One just adds a constant $0.6 \mathrm{~nm}$ to the pore size calculated by standard BJH. This constant shift basically does not change our conclusion in Fig. 7. With a single parameter, we could still predict the pore size versus wall thickness plot fairly well.

\subsection{Increased hydrothermal stability}

Ryoo and coworkers [29,30] have demonstrated prolonged hydrothermal treatment of as-synthesized materials in salt solutions at $100{ }^{\circ} \mathrm{C}$ could increase its hydrothermal stability. From the present study, the thickening of the wall of MCM-41 maybe the major cause of the salt stabilization effect. It seems that treating at the higher temperature $150{ }^{\circ} \mathrm{C}$ will enhance further the salt stabilization effect.

\section{Conclusions}

We have shown in this paper that the physical chemistry in the problem of post-synthesis hydrothermal effect on the pore size of MCM-41 can be systematically approached. We studied the wellcontrolled system of treating samples in neutral water or salt solutions. The pore expands upon increasing temperature to $150{ }^{\circ} \mathrm{C}$ with simultaneous wall thickening. This is explained in terms of water penetration. Evidence from TGA studies is provided to support this explanation. However, hydrothermal treatment in mother liquor with base conditions and supply of source materials would give one larger pore expansion. This is due to the decomposition of larger amounts of surfactant to amine to swell the micelles.

The clear inverse relationship between wallthickness and pore size can be fitted by a simple model of local adjustment of the silica wall to the change of surfactant content. The model is based on conservation of silica materials and channels and the adjustment is continuous and local. The adjustment of the surfactant content in the MCM41 composite can be finely controlled by using various salt solutions in hydrothermal treatment. This is based on the well-known ion-surfactant binding strength that follows the Hofmeister series.

This work is part of a series of study of the physical chemistry of the changes that is associated in the synthesis and post-synthesis treatment of mesoporous materials [26,28,40]. It is demonstrated that the physicochemical process can be studied in a quantitative way to understand the fundamental thermodynamic and kinetic principle involved in the making of the complex periodical organic/inorganic composite.

\section{Acknowledgements}

This research was supported by the China Petroleum Co. and the National Science Council of Taiwan (NSC 88-2113-M-002-027). 


\section{References}

[1] (a) C.T. Kresge, M.E. Leonowicz, W.J. Roth, J.C. Vartuli, J.S. Beck, Nature 359 (1992) 710;

(b) J.S. Beck, J.C. Vartuli, W.J. Roth, M.E. Leonowicz, C.T. Kresge, K.D. Schmitt, T.W.C. Chu, D.H. Olson, E.W. Sheppard, S.B. Higgins, J.L. Schlenker, J. Am. Chem. Soc. 114 (1992) 10834.

[2] K.J. Edler, J.W. White, Chem. Mater. 9 (1997) 1226.

[3] A. Sayari, M. Kruk, M. Jaroniec, I.L. Moudrakovski, Adv. Mater. 10 (1998) 1376.

[4] F. Di Renzo, F. Testa, J.D. Chen, H. Cambon, A. Galarneau, D. Plee, F. Fajula, Micropor. Mesopor. Mater. 28 (1999) 437.

[5] C.Y. Mou, H.P. Lin, Pure Appl. Chem. 72 (2000) 137.

[6] Q. Huo, D.I. Margolese, G.D. Stucky, Chem. Mater. 8 (1996) 1147.

[7] A.F. Gross, E.J. Ruiz, S.H. Tolbert, J. Phys. Chem. B 104 (2000) 5448.

[8] D.F. Juan, E. Ruiz-Hitzky, Adv. Mater. 12 (2000) 430.

[9] D. Khushalani, A. Kuperman, G.A. Ozin, K. Tanaka, J. Garces, M.M. Olken, N. Coombs, Adv. Mater. 7 (1995) 842.

[10] M. Kruk, M. Jaroniec, A. Sayari, Micropor. Mesopor. Mater. 27 (1999) 217.

[11] M.S. Morey, S. O’Brien, S. Schwarz, G.D. Stucky, Chem. Mater. 12 (2000) 898.

[12] Z. Luan, W. Zhou, J. Klinowski, J. Chem. Soc. Faraday Trans. 94 (1998) 979.

[13] (a) C.F. Cheng, W. Zhou, Z.J. Klinowski, Chem. Phys. Lett. 263 (1996) 247;

(b) C. Cheng, W. Zhou, D.H. Park, J. Klinowski, M. Hargreaves, L.F. Gladden, J. Chem. Soc. Faraday Trans. 93 (1997) 359;

(c) W.Z. Zhou, J. Klinowski, Chem. Phys. Lett. 292 (1998) 207.

[14] A. Corma, Q. Kan, M.T. Navarro, J. Perez-Pariente, F. Rey, Chem. Mater. 9 (1997) 2123.

[15] A. Sayari, P. Liu, M. Kruk, M. Jaroniec, Chem. Mater. 9 (1997) 2499.

[16] L. Chen, T. Horiuchi, Y. Mori, K. Maeda, J. Phys. Chem. B 103 (1999) 1216.

[17] (a) R. Ryoo, J.M. Kim, J. Chem. Soc., Chem. Comm. (1995) 711 ;

(b) R. Ryoo, S. Jun, J. Phys. Chem. B 101 (1997) 317.

[18] X. Chen, L. Huang, Q. Li, J. Phys. Chem. B 101 (1997) 8460.

[19] R. Ryoo, J.M. Kim, J. Chem. Soc., Chem. Commun. (1995) 711.
[20] D. Das, C.M. Tsai, S. Cheng, Chem. Commun. (1999) 473.

[21] R. Mokaya, J. Phys. Chem. B 103 (1999) 10204.

[22] T. Blasco, A. Corma, A. Martinez, P. Martinez-Escolano, J. Catal. 77 (1998) 306.

[23] V.I. Srdanov, I. Alxneit, G.D. Stucky, C.M. Reaves, S.P. DenBaars, J. Phys. Chem. B 102 (1999) 3341.

[24] M. Kruk, M. Jaroniec, A.J. Sayari, Phys. Chem. B 103 (1999) 3651.

[25] M. Kruk, M. Jaroniec, A. Sayari, J. Phys. Chem. B 103 (1999) 4590.

[26] H.P. Lin, C.P. Kao, C.Y. Mou, J. Phys. Chem. B 104 (2000) 7885.

[27] D.N. Rubingh, P.M. Holland, Cationic Surfactant: Physical Chemistry, in: Surfactant Science Series, vol. 37, Marcel Dekker, New York, 1991.

[28] H.P. Lin, C.P. Kao, C.Y. Mou, Micropor. Mesopor. Mater. 48 (2001) 135.

[29] R. Ryoo, J.M. Kim, C.H. Ko, C.H. Shin, J. Phys. Chem. 100 (1996) 17718.

[30] J.M. Kim, S. Jun, R. Ryoo, J. Phys. Chem. B 103 (1999) 6200.

[31] A. Corma, D. Kumar, Stud. Surf. Sci. Catal. 117 (1998) 201.

[32] H.P. Lin, S. Cheng, C.Y. Mou, Micropor. Mater. 10 (1997) 111.

[33] (a) H.P. Lin, S.B. Liu, C.Y. Mou, Chem. Lett. 12 (1999) 1341 ;

(b) H.P. Lin, C.Y. Mou, S.B. Liu, Adv. Mater. 12 (2000) 103.

[34] R.K. Iler, The Chemistry of Silica, John-Wiley, New York, 1979.

[35] C.J.G. Van der Grift, J.W. Geus, H. Barten, R.G.I. Leferink, J.C. van Miltenburg, A.T. den Ouden, in: K.K. Unger et al. (Eds.), Characterization of Porous Solids, Elsevier, Amsterdam, 1988

[36] L. Gaillon, J. Lelievere, R.J. Gaboriaud, Coll. Interf. Sci. 213 (1999) 287.

[37] K. Hayakawa, T. Morita, M. Ariyoshi, T. Maeda, I.J. Aatake, Coll. Interf. Sci. 177 (1999) 621.

[38] (a) M. Kruk, M. Jaroniec, A. Sayari, J. Phys. Chem. B 101 (1997) 583;

(b) G.P. Jaroniec, M. Kruk, M. Jaroniec, J. Phys. Chem. B 103 (1999) 10670;

(c) M. Kruk, M. Jaroniec, A. Sayari, Chem. Mater. 11 (1999) 492.

[39] H.Y. Zhu, G.Q. Lu, J. Phys. Chem. B 102 (1998) 7371.

[40] H.P. Lin, S.T. Wong, C.Y. Mou, C.Y. Tang, J. Phys. Chem. B 104 (2000) 8967. 